\title{
WordTree: Results of a Word Prediction System Presented Thanks to a Tree
}

\author{
Georges Badr and Mathieu Raynal \\ Université Paul Sabatier, IRIT \\ 31062 Toulouse cedex, France \\ \{badr, raynal\} @irit.fr
}

\begin{abstract}
For mobility and portability reasons, written communication devices are getting smaller and physical keyboards are replaced with virtual or onscreen one (PDA, pocket pc, GPS, etc.). Text entry has become a tiring job for people (ordinary and motor disabled people) because of the low entry speed. A list of predicted words can be presented to reduce the number of clicks to complete the word. However, these techniques present a disadvantage: let's consider the situation where the word the user wants to enter is not presented in the list. He has then to continue his writing. The prediction system narrows the possibilities in order to display the wanted word. In this paper we present a novel interaction technique in which the user can select a substring of the word. The user clicks on any letter, and the substring to this letter is inserted in the text. We also present the simulated experiments to compare our system to the classical list. The results are then discussed and analyzed.
\end{abstract}

\section{Introduction}

Nowadays, with the expansion of the Web and the appearance of the mobile computing and technology with all its services, the communication has become more and more one of the necessaries (SMS, mail, chat, forums, etc.). Text entry is then presented as the most used service with computers. Therefore, keyboards became one of the most important interacting elements in computing. Mobile devices are more and more smaller and are losing their physical keyboards. Virtual or on-screen keyboards ${ }^{1}$ are being used instead (PDA, pocket pc, GPS, etc.). Text is entered by a single pointing device such as a mouse or stylus.

Moreover, people suffering from motor disabilities in their upper members (myopathy ${ }^{2}$, cerebral palsy ${ }^{3}$, musculoskeletal disorders, etc.), have difficulties using physical keyboards and thus they are obliged to use soft keyboards. Due to their disabilities, they cannot easily move their hands or fingers, neither the pointing device,

\footnotetext{
${ }^{1}$ Virtual keyboard or on-screen keyboard, also known as software keyboard, is a system that replaces the hardware keyboard on a computing device by its numerical representation. The text entry is done on these keyboards using a pointing device (mouse, stylus...).

${ }^{2}$ In medicine, a myopathy is a neuromuscular disease in which the muscle fibers do not function.

${ }^{3}$ Cerebral injuries causing physical disability in human development.
} 
through the keys of the keyboards causing a low text entry speed. This repeated move is so tiring and annoying for them.

To remedy these shortcomings concerning the slow text entry and the tiredness due to the "large" distance between letters, we propose to improve the speed of entry and increase keyboards' performance. Our aim is to minimize the pointing time and the movement between keys, as well as to reduce the number of clicks one has to do in order to complete their word. For this purpose, consistent efforts have already been made using assistive aids allied to the text entry system. Those aids are like add-ins on the standard keyboards: prediction systems are allied with interacting techniques to help users to reduce the number of operations they would do on original keyboards (i.e. number of clicks) leading for maximizing the entry speed and performance.

One technique used to speed up text entry is to present a list of "best guesses" (predicted words) for the word the user is currently entering. The user can select the word that continues the prefix he has already entered. As the user continues to enter letters, the system updates the list of word predictions to conform the user's input. But performance on such systems is not satisfactory because the user is obliged to select the whole word. Furthermore, if the aimed word is not presented in the list, the user has to continue his text entry to increase the probability of his word so it can be shown with the guessed words in the list.

WordTree is a novel interaction technique based on a list of predicted words where the user can select a substring of the word. Select the whole word from the list is not necessary, but the user can click on any character of the word, and the substring to this letter is inserted in the text. For example if the user wants to write "applicable", after typing "app" on the keyboard, the word "application" is displayed in the list, the user can select "applica" to speed his entry.

After a brief background of the word prediction list, we describe the principle of our system. Then, we present a first evaluation based on a simulation with an optimal use of our system and compare its results with a same simulation carried out with a classical list of word prediction. Finally we discuss the first results of this evaluation/comparison.

\section{Background on Lists of Prediction}

Designing communication aids is a challenging and ambitious research area. Engineers and programmers design systems in order to speed up the text entry. Ones of the most famous methods are the word predictions which implement Artificial Intelligence, Natural Language Processing, Machine Learning, etc. Word prediction is one of the communication aids that are allied to a software keyboard or a text entry system. The aim is to predict or guess the word that the user is entering in order to complete it. This can speed up text entry by reducing the number of clicks the words would need to be completed.

Word prediction systems can de divided into two categories: integrated completion (the predicted word is directly inserted in the text as VITIPI [1]) and a list of candidate predicted words. For this latter category of word prediction systems, each time, a list of the most probable words for the current user's input is displayed in a specific zone. The user goes in the list and searches if the aimed word is predicted and selects 
it. The word is then inserted into the text. Although the user needs one additional click which is the selection click, those systems can reduce the total number of operations the user has to do in order to complete his word. Different systems implement the prediction list, varying the size, the position, the number of candidate words or the form of the list.

Some systems like Sibylle [2], HandiAS [3] are software keyboards using word prediction to aid the communication. Predicted words are displayed in a fixed list from which the user can pick his word to complete his prefix. Keystrokes [4] proposes the words in two lists (cf. Fig. 1): one horizontal presented above the keyboard keys and another one vertical that can be placed anywhere on the screen and be resized as needed. Cognitive researches and especially Miller's works [5] indicate that it's easy for human to search and remember 7 words at a time. Thus, two lists in one system may cause a large cognitive charge for the user.

\begin{tabular}{|c|c|c|c|c|c|c|c|c|c|c|c|c|c|c|c|c|}
\hline$\theta 0$ & & & & & $\begin{array}{l}\text { Keyst } \\
\text { ent }\end{array}$ & Tokes & (trial cc & opy. 1 & 5 days & to exp & & & & & 0 & 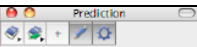 \\
\hline 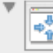 & - 6 & 管 & $\theta \sqrt{2}$ & 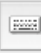 & 3 & 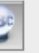 & 8 & $\because$ & $i$ & & $-\mathbb{N}$ & A & $\vec{v}$ & & ( & [Texe- shortens \\
\hline s: & & & & & & & & & & & & & & & 16a & and \\
\hline \begin{tabular}{l|l} 
\\
\end{tabular} & and & had & & e & his & 1 & in & it & of & that & & the & to & was & you & hac \\
\hline$\S$ & 1 & 2 & 3 & 4 & 5 & 6 & 7 & 8 & 9 & 0 & - & $=$ & $凶$ & 8 & 00 & $\begin{array}{l}\text { ne } \\
\text { his } \\
\text { hes }\end{array}$ \\
\hline$\rightarrow$ & q & $w$ & e & r & $\mathrm{t}$ & $y$ & u & i & 0 & $p$ & [ & ] & ה & 91 & П & in \\
\hline 슴 & a & 5 & d & $f$ & g & $\mathrm{h}$ & $\mathrm{j}$ & k & I & ; & ' & 1 & & - & & of \\
\hline 仓 & ` & $z$ & $x$ & C & $v$ & b & $\mathrm{n}$ & $\mathrm{m}$ & , & . & 1 & $\hat{\imath}$ & $\uparrow$ & + & $\pi$ & $\begin{array}{l}\text { that } \\
\text { the }\end{array}$ \\
\hline$\wedge$ & $\tau$ & मै & & & & & & & & $\kappa$ & 4- & $\rightarrow$ & $\vdots$ & $x$ & 回 & $\begin{array}{l}\text { to } \\
\text { was } \\
\text { with }\end{array}$ \\
\hline & & & & & & & & $\mathbf{A}$ & & & & & & & & you \\
\hline
\end{tabular}

Fig. 1. Keystrokes: A) one list horizontal on the top of the keyboard; B) one vertical on the right

Other systems, like Pen-Operation Based On eXample - PoBox [6] displays a list of candidate words (a pull-down or popup menu) near the cursor. The idea is to minimize the distance between the letter already clicked and the complete word the system predicts. But, on the other hand, the list hides an important part of the keyboard and if the next letter to enter is under the list, the user can be annoyed by the list. FASTY [7], Dicom [8], Premier Predictor Pro - P3 [9] and other commercial systems, pop up a list near the writing cursor in the text. Those are not software keyboards but display only candidate words to complete the text.

In all these systems, the user has to select the whole word. For this reason, he has to complete the text entry, so the system updates the list of words until the desiredword is displayed in the list. Otherwise, he has to complete the word manually. In this case, the user will lose time, not only for searching the word from the list, but also to return to the text to continue his typing. The main goal for prediction systems - reducing time, keystrokes and tiredness is not reached. To remedy this shortcoming, we have implemented WorldTree, a new interaction system with which the user can select a part of the word in the list, and insert the select substring in the text. 


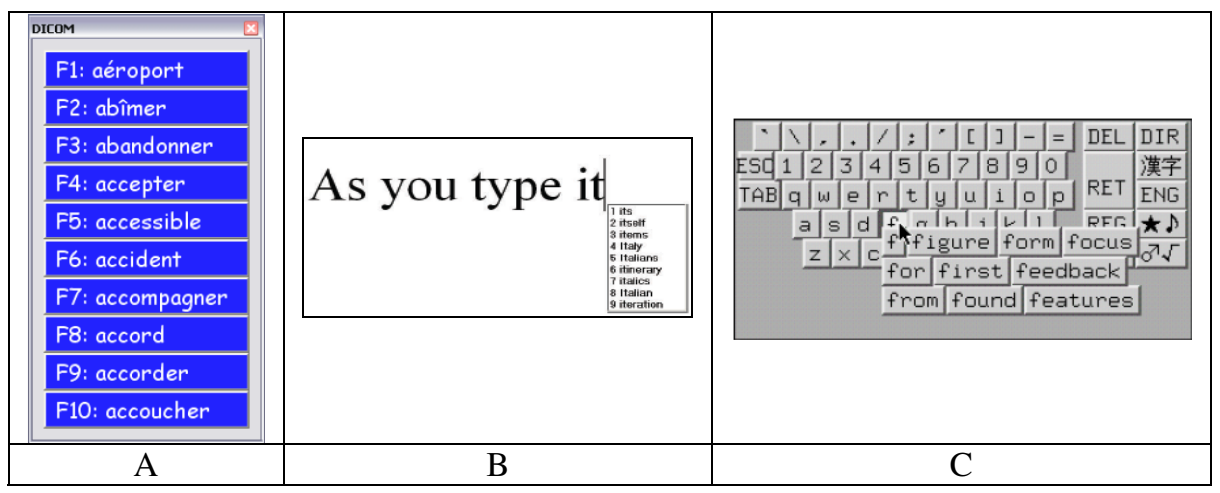

Fig. 2. A) Prediction list of the Dicom ; B) the P3 ; C) PoBox

\section{WordTree System}

As mentioned in [10] word prediction lists aim to accelerate the text entry speed by reducing the number of clicks (keystrokes) required to enter text one should do on an ordinary keyboard to enter the same text. This can also reduce the number of errors the user may commit during his writing. However, those systems can reduce the speed of writing and may annoy the user. This shortcoming is caused by the fact that the user has to select a whole word from the list.

\subsection{Principles}

Considering the situation where the aimed word is not present in the list, the user has then to continue his writing waiting for his word to come out and be shown in the list, and sometimes he has to complete his word manually. The idea is to design a new interaction with the prediction list in a way that allows the user to select a substring of the word without being forced to select the whole word. In this way, the user is not obliged to complete his word manually is order to make it appear in the list: once any other word is listed and contains a part of his word, he can select this substring so he can accelerate his typing.

Each character is represented by a clickable "node". If a node is clicked, the system validates all the nodes that are among the root of the word and the node clicked. While typing a text, and once any other word is listed and contains a part of what the user is entering, he can click on any node so he can select (cf. Fig. 3) the substring that may complete the best what he is writing. That may accelerate his typing. For example, if the user wants to write the word "applicable", he begins by typing "app" on the keyboard. A list of candidate words is then displayed for him in a form of a tree. For some reason (i.e. low probability for this word in the prediction system), suppose that the word "applicable" is not listed yet, instead we have the word "application". In order to go forward in his text the user can click on the second "a" of "application" and the substring "applica" is inserted in the text. By this manner the user would accelerate his typing without the need to continue the typing and waiting for the wanted word to come out. 


\section{BOMB ARDE \\ BOUCHE \\ BOU CLE \\ BOU I L LON \\ BOULEVERSE \\ BOURRE \\ BOUTONNE \\ BOYCOT T}

Fig. 3. WordTree interface

\subsection{Word Prediction System Used}

The WordTree interaction presents to the user a list of words with the prefix that he began to type. In order to be able to guess or predict the word, WordTree is allied to a word prediction system based on a lexicographic tree constructed from a set of words (dictionary). Each word is represented by a path from the root to a leaf of the tree, where each character is a node of the tree (cf. Fig. 4). Two words with the same prefix use the same initial path to avoid creating too many new nodes. For words which are prefixes for other words, a special leaf "end word" was created.

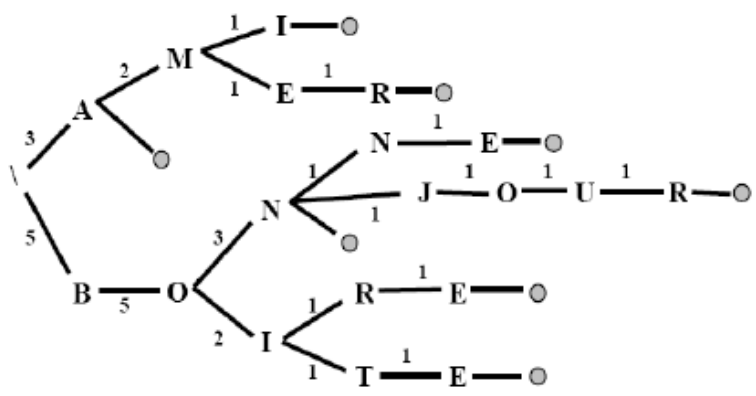

Fig. 4. Lexicographic tree done with the following French words: a, ami, amer, bon, bonne, bonjour, boire, boite, extracted from [11]

We chose to give weight to each arc of the tree. At each added word, the arcs between nodes which are crossed are incremented by 1 . This system takes into account the beginning of the word which has just been entered by crossing the tree according to what was already entered. With regard to this beginning of word, it classifies the characters that can follow it according to their probability of appearance (the higher the value of the arc is the greater the character's chance of appearing will be). This 
weight will let it sort the predicted words according to their probability defined by their weights.

The lexicographic tree guesses, for every entered letter, the set of possible following characters completing what has been already entered.

\section{Experiments}

Our hypothesis is that with this type of interaction, the user can use more regularly the result of prediction system by choosing a piece of word.

\subsection{Evaluation by Simulation}

Before testing our system with users, we wanted to determine the a priori performance of this interaction by carrying out a theoretical simulation. This simulation consists of testing all possible cases of text entering with and without WordTree. The results would inform us on the performance of the system.

Protocol. Our first experiment aimed to determine the theoretical performance of our system. For this purpose, we simulated the optimal use of the two systems: WordTree and the classical list of word prediction. This simulation enables us to find a limit under of the user's performance with our system. It consists in keyboarding the set of words in Scrabble ("Officiel du Scrabble") composed by 364,370 French words and $3,641,140$ characters. This simulation is called "perfect" because if the WordTree system or the other classical list of word prediction can be used, we choose this one and not the character on keyboard layout. Note that WordTree, as well as the classical system, are using the same prediction algorithm described in paragraph 3.2.

In order to determine the performance of our interaction paradigm we tried to compare it to a classical system using the ordinary word selection from a prediction list. To be able to evaluate our system we measured two variables:

1. The KeyStroke Per Character (KSPC) [12] which is "the number of keystrokes (or taps in case the pointing is by a stylus) required, on average, to generate a character of text for a given text entry technique in a given language". This metric is calculated by dividing the total number of operations (taping, clicking...) the user has done, including the error correction, to the total number of characters in the word. Thus KSPC can be defined as follows:

$$
\mathrm{KSPC}=\frac{\text { total number of operations }}{\text { number of characters }}
$$

2. The Hit Rate (HR) [13] which evaluates the percentage of times where the "aimed" is listed. Since our system allows the user to select a part of a predicted word, we can redefine the HR as the number of times the user clicks on a node to select the substring that could complete his word. For example, if the word does not exist in the list, but only a part of it, then, with our system the user can select this part, that was impossible with the classical list of predicted words.

$$
\mathrm{HR}=\frac{\text { number of time the user uses the prediction }}{\text { total number of time words are presented }} \times 100
$$


Results. The results of this simulation show a significant progress in performance compared to a classical word prediction list. In fact, we have obtained a decrease of about $22 \%$ in the KSPC and an increase of $75 \%$ in the HR. Thus, theoretically, in order to write a word, the user would have to do less clicks and he would use more frequently the predicted words from the list. Table 1 and Fig. 5 show the results we obtained from our first experiment.

Table 1. Comparison of KSPC and HR for normal keyboard, a classical list and WordTree

\begin{tabular}{lll}
\hline Writing assistance & KSPC & HR \\
\hline Normal Keyboard & 1 & - \\
Classical prediction list & 0.92 & $3.7 \%$ \\
WordTree & 0.71 & $18.7 \%$ \\
\hline
\end{tabular}

Theoretically, our system has reached its goal by accelerating text entry and reducing the fatigue. Thus, by decreasing the number of clicks or KSPC, the system would reduce the number of operations the user has to do, and thus reduce his fatigue. On the other hand the hit rate indicates that the user would use more the predicted list, and therefore, this would reduce the typing error.

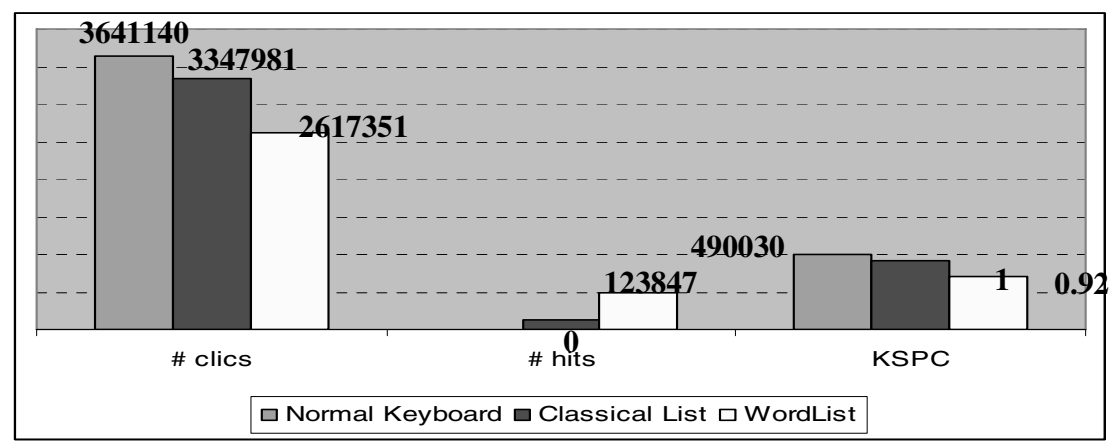

Fig. 5. Comparing the number of clicks, the number of hits and the KSPC of the 3 types of typing systems

As the predictive process does not take into account the searching time for the character among the KeyGlasses, we conducted an evaluation with a disabled motor subject to verify our hypotheses.

\subsection{Evaluation with a Disabled Motor Subject}

Protocol. The experiment was conducted on a laptop HP running Microsoft Windows XP. The subject used a trackball to interact with the soft keyboard. We chose for this experiment to restrict us to a soft keyboard which contained only the 26 characters of the Latin alphabet and the space bar. The soft keyboard was developed with Java (cf. Fig. 6). 


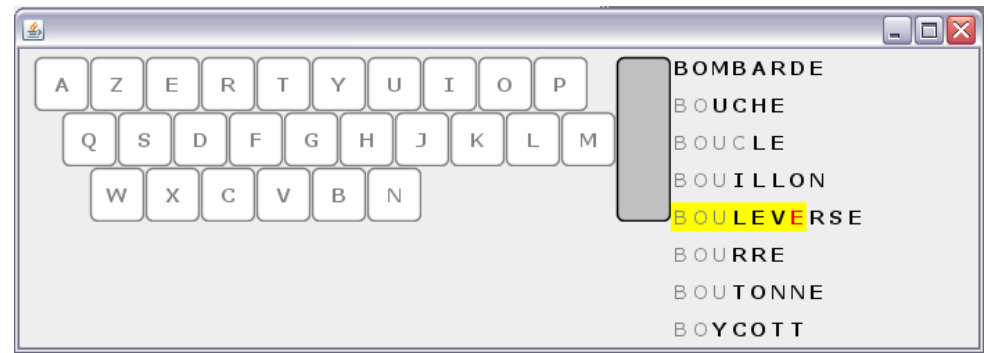

Fig. 6. SofKeyboard used

He performed two exercises of word copy: one exercise with the AZERTY keyboard with classical list of word prediction, and the other with the WordTree system. For each exercise, the subject had to copy out 22 words (157 characters), which were the same for the two exercises. Words were chosen as the most usually used in French and as to represent a maximum of different co-occurrences.

The word to be copied was presented on a line, and the word being typed by the user appeared on the line below. The text entry errors were not displayed on the screen. Instead there was a visual and audio feedback signaling the error and the strip did not move until the subject entered the right character. At the end of each word, the participant had to hit the space bar.

Results. The participant entered the 22 words in 342.6 seconds and 151 hits (that is to say $0.46 \mathrm{cps}$ and $\mathrm{KSPC}=0.96$ ) with the soft keyboard with the classical list of word prediction, whereas he needed only 324.7 seconds and 104 hits $(0.48 \mathrm{cps}$ and $\mathrm{KSPC}=0.66$ ) with the WordTree system. Thus he gain $5,2 \%$ of time with the WordTree relatively to the standard list of word prediction. During the exercises, when the current character differed from the expected character, the error was recorded. The number of errors was 1 for the soft keyboard with the list of word prediction and 2 with the WordTree system.

\section{Conclusion}

In this article, we suggest a new interaction to use a list of predicted words: the user can click on any character of a word in the list and then select a substring of this word. The first results reported by our experiments show that this interaction can be beneficial for text entry speed compared to a classical list.

\section{References}

1. Boissière, P., Dours, D.: VITIPI: versatile interpretation of text input by persons with impairments. In: Proc. 5th International Conference on Computers for Handicapped Persons, ICCHP 1996, Linz, Autriche, pp. 165-173 (1996)

2. Wandmacher, T., Antoine, J.-Y., Poirier, F., Departe, J.-P.: SIBYLlE, An Assistive Communication System Adapting to the Context and Its User. ACM Transactions on Accessible Computing 1(1), 1-30 (2008) 
3. Maurel, D., Le Pevedic, B., Rousseau, O.: The Syntactic Prediction with Token Automata: Application to HandiAS System. In: Champarnaud, J.-M., Maurel, D., Ziadi, D. (eds.) WIA 1998. LNCS, vol. 1660, p. 100. Springer, Heidelberg (1999)

4. Keystrokes on-screen keyboard, user manual, http: / / www.assistiveware.com/files/KeyStrokesXmanualA4.pdf

5. Miller, G.: The Magical Number Seven, Plus or Minus Two. The Psychological Review 63(2), 81-97 (1956)

6. Masui, T.: An efficient text input method for pen-based computers. In: Proceedings of the ACM Conference on Human Factors in Computing Systems CHI 1998 (April 1998)

7. Beck, C., Seisenbacher, G., Edlemayer, G., Zagler, W.L.: First User Test Results with the Predictive Typing System FASTY

8. Programme développé par Gwénaëlle Marcou et Christophe Litzinger en collaboration avec Handicap International - Centre Icom' - (Mode d'emploi)

9. Premier Literacy, http: / / www . readingmadeeasy. com

10. Koester, H., Levine, S.: The effect of a word prediction feature on user performance. Augmentative and Alternative Communication 12(3), 155-168 (1996)

11. Raynal, M.: Systèmes de saisie de textes pour les personnes handicapées moteur: optimisation, interaction et mesure de l'utilisabilité, Ph.D thesis, IRIT (2005)

12. MacKenzie, I.S.: KSPC (Keystrokes per Character) as a Characteristic of Text Entry Techniques. In: HCI mobile 2002 (2002)

13. Fazly, A., Hirst, G.: Testing the efficacy of part-of-speech information in word completion. In: Proc. Workshop on Language Modeling for Text Entry Methods, EACL 2003, Budapest, Hongrie (2003) 